\title{
Chaos Control of a Chemical Chaotic System via Time-delayed Feedback Control Method
}

\author{
Chang-Jin $\mathrm{Xu}^{1} \quad \mathrm{Yu}-\mathrm{Sen} \mathrm{Wu}^{2}$ \\ ${ }^{1}$ Guizhou Key Laboratory of Economics System Simulation, Guizhou University of Finance and Economics, Guiyang 550004, China \\ ${ }^{2}$ School of Mathematics and Statistics, Henan University of Science and Technology, Luoyang 471023, China
}

\begin{abstract}
In this paper, the control of chemical chaotic dynamical system is investigated by time-delayed feedback control technique. The controllability and the stability of the equilibriums and local Hopf bifurcation of the system are verified. Some numerical simulations which show the effectiveness of the time-delayed feedback control method are provided.
\end{abstract}

Keywords: Chemical system, chaos, stability, Hopf bifurcation, time delay.

\section{Introduction}

In recent years, chaos in control systems and controlling chaos in dynamical systems have both attracted growing attention ${ }^{[1]}$. A chaotic system has complex dynamical natures such as excessive sensitivity to initial conditions, broad spectrums of Fourier transform, and fractal properties of the motion in phase space ${ }^{[2]}$. Due to its powerful applications in power conversion, chemical reactions, biological systems, information processing and secure communications, etc. ${ }^{[1]}$, chaos control has seen a great deal of research activities from various fields ${ }^{[3-9]}$. Chaos is sometimes undesirable. In many cases, we wish to avoid and eliminate such behaviors. Recently there are numerous techniques to control chaos such as method presented by Ott et al. ${ }^{[3]}$, active control ${ }^{[10]}$, observer-based control ${ }^{[11]}$, feedback and non-feedback control ${ }^{[11-14]}$, inverse optimal control $^{[15]}$, adaptive control ${ }^{[16,17]}$, etc. In 2005, Huang and Yang ${ }^{[18]}$ found a chaotic attractor from the following chemical system

$$
\left\{\begin{array}{l}
\dot{x}_{1}=\alpha_{1} x_{1}-k_{-1} x_{1}^{2}-x_{1} y_{1}-x_{1} z_{1} \\
\dot{y}_{1}=x_{1} y_{1}-\alpha_{5} y_{1} \\
\dot{z}_{1}=\alpha_{4} z_{1}-x_{1} z_{1}-k_{-5} z_{1}^{2}
\end{array}\right.
$$

where $\alpha_{1}, \alpha_{4}, \alpha_{5}, k_{-1}$ and $k_{-5}$ are all positive constants. When $\alpha_{1}=30, \alpha_{4}=16.5, \alpha_{5}=10, k_{-1}=0.415$ and $k_{-5}=0.5$, system (1) is chaotic (see Fig. 1 ).

The main objective of this paper is to guide the chaotic trajectories for a chemical system to unstable equilibrium points or unstable periodic orbits by applying the feedback control technique. In contrast to the formal control methods mentioned above, since the formal control methods are based on the invariant manifold structure of unstable orbits, the feedback control method is more simple and convenient to control chaos in continuous dynamical systems. Thus it

\footnotetext{
Regular paper

Manuscript received May 12, 2013; revised September 11, 2013

This work was supported by National Natural Science Foundation of China (Nos. 11261010 and 11101126), Soft Science and Technology Program of Guizhou Province (No. 2011LKC2030), Natural Science and Technology Foundation of Guizhou Province (No. J[2012]2100), Governor Foundation of Guizhou Province (No. [2012]53) and Doctoral Foundation of Guizhou University of Finance and Economics.
}

is theoretically well understood, but difficult to apply to
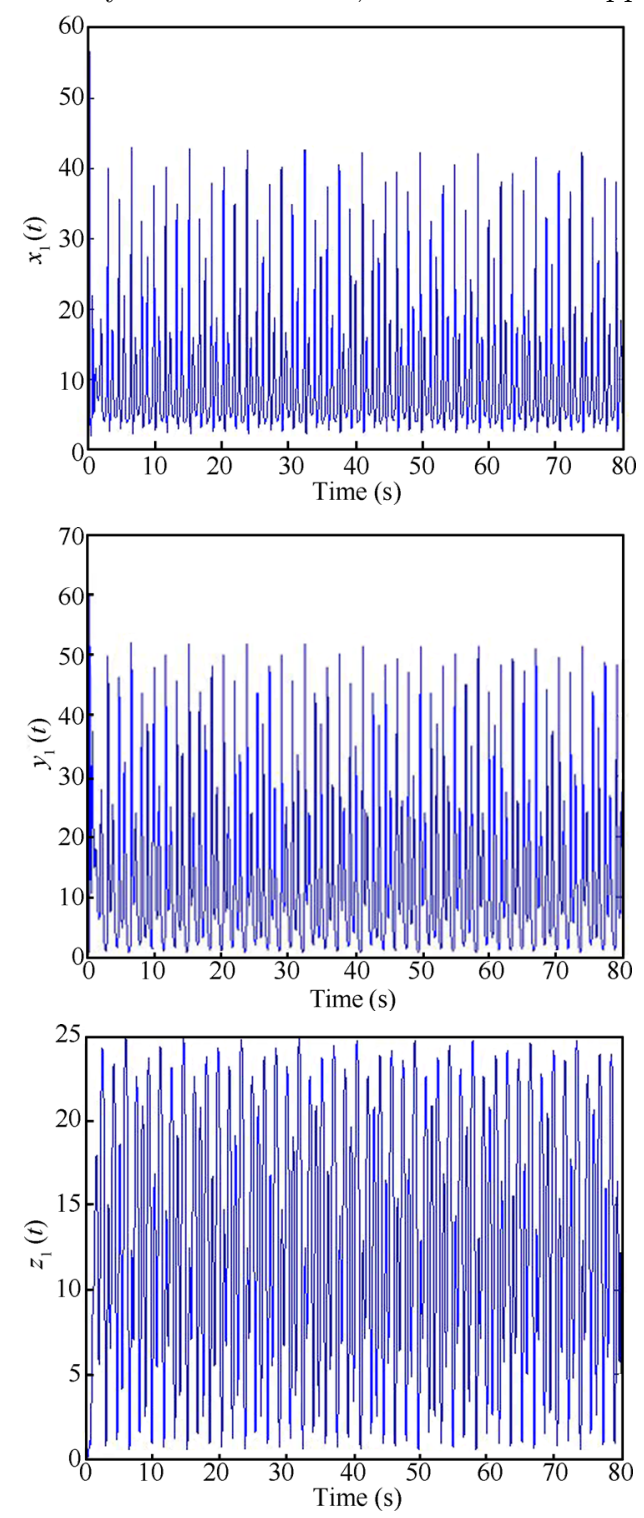


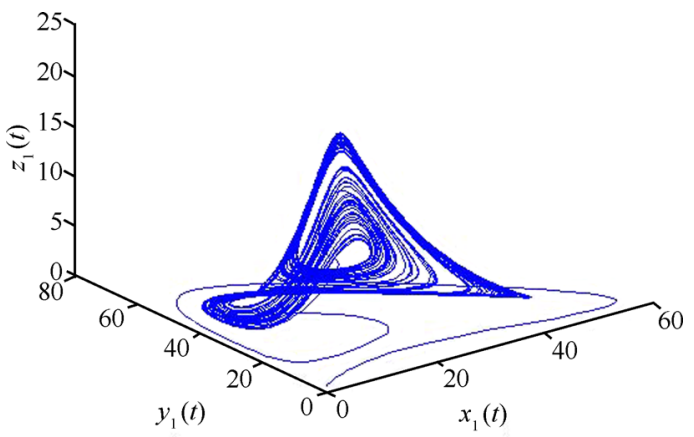

Fig. 1 The chaotic attractor of system (1) with $\alpha_{1}=30, \alpha_{4}=$ $16.5, \alpha_{5}=10, k_{-1}=0.415$ and $k_{-5}=0.5$.

fast experimental systems. In addition, we shall point out that although there are many papers which deal with the problem of chaos control, very few focus on chemical chaotic systems. We believe that the investigation into chaos control of the chemical system can give some information for practical design and achieve better performance in some specific applications.

\section{Controlling chaos}

In this section, we shall apply the conventional feedback technique to the dynamical system (1). Our aim is to drag the chaotic trajectories to the equilibrium or periodic orbits. Following the idea of Pyragas ${ }^{[19]}$, we add a time-delayed force $k\left[x_{1}(t)-x_{1}(t-\tau)\right]$ to the first equation of system (1), then system (1) takes the form

$$
\left\{\begin{aligned}
\dot{x}_{1}= & \alpha_{1} x_{1}-k_{-1} x_{1}^{2}-x_{1} y_{1}-x_{1} z_{1}+ \\
& k\left[x_{1}-x_{1}(t-\tau)\right] \\
\dot{y}_{1}= & x_{1} y_{1}-\alpha_{5} y_{1} \\
\dot{z}_{1}= & \alpha_{4} z_{1}-x_{1} z_{1}-k_{-5} z_{1}^{2}
\end{aligned}\right.
$$

where $k$ is a real constant. It is easy to see that if the conditions

$$
\alpha_{4}>\alpha_{5},\left(\alpha_{1}-k_{-1}\right) k_{5}>\alpha_{4}-\alpha_{5}
$$

are satisfied, then system (2) has a unique positive equilibrium $E\left(x_{1}^{*}, y_{1}^{*}, z_{1}^{*}\right)$, where

$x_{1}^{*}=\alpha_{5}, y_{1}^{*}=\frac{\left(\alpha_{1}-k_{-1} \alpha_{5}\right) k_{5}-\alpha_{4}+\alpha_{5}}{k_{-5}}, \quad z_{1}^{*}=\frac{\alpha_{4}-\alpha_{5}}{k_{-5}}$.

Linearized system of $(2)$ around $E\left(x_{1}^{*}, x_{2}^{*}, x_{3}^{*}\right)$ takes the form

$$
\left\{\begin{aligned}
& \dot{x}_{1}=\left(\alpha_{1}+k-2 k_{-1} x_{1}^{*}-y_{1}^{*}-z_{1}^{*}\right) x_{1}- \\
& x_{1}^{*} y_{1}-x_{1}^{*} z_{1}-k x_{1}(t-\tau) \\
& \dot{y}_{1}= y_{1}^{*} x_{1}+\left(x_{1}^{*}-\alpha_{5}\right) y_{1} \\
& \dot{z}_{1}=-z_{1}^{*} x_{1}+\left(\alpha_{4}-x_{1}^{*}-2 k_{-5} z_{1}^{*}\right) z_{1}
\end{aligned}\right.
$$

whose characteristic equation is given by

$$
\lambda^{3}+m_{2} \lambda^{2}+m_{1} \lambda+m_{0}+\left(n_{2} \lambda^{2}+n_{1} \lambda+n_{0}\right) \mathrm{e}^{-\lambda \tau}=0
$$

where

$$
\begin{aligned}
m_{0}= & 2 k_{-5} z_{1}^{*}\left(x_{1}^{*}-\alpha_{5}\right)\left(a_{1}+k-2 k_{-1} x_{1}^{*}-y_{1}^{*}-z_{1}^{*}\right)- \\
& x_{1}^{*} z_{1}^{*}+x_{1}^{*} y_{1}^{*}+x_{1}^{*} z_{1}^{*}\left(x_{1}^{*}-\alpha_{5}\right)+2 k_{-5} x_{1}^{*} y_{1}^{*} z_{1}^{*}- \\
& x_{1}^{*} y_{1}^{*}\left(x_{1}^{*}-\alpha_{4}\right) \\
m_{1}= & \left(a_{1}+k-2 k_{-1} x_{1}^{*}-y_{1}^{*}-z_{1}^{*}\right)\left(x_{1}^{*}-\alpha_{5}\right)- \\
& 2 k_{-5} z_{1}^{*}\left(a_{1}+k-2 k_{-1} x_{1}^{*}-y_{1}^{*}-z-1^{*}\right)- \\
& x_{1}^{*} z_{1}^{*}+x_{1}^{*} y_{1}^{*}-2 k_{-5} z_{1}^{*}\left(x_{1}^{*}-\alpha_{5}\right) \\
m_{2}= & -\left[a_{1}+k-\left(2 k_{-1}-1\right) x_{1}^{*}-y_{1}^{*}-\right. \\
& \left.\left(1+2 k_{-5}\right) z_{1}^{*}-\alpha_{5}\right] \\
n_{0}= & -2 k k_{-5} z_{1}^{*}\left(x_{1}^{*}-\alpha_{5}\right) \\
n_{1}= & k\left(2 k_{-5} z_{1}^{*}-x_{1}^{*}+\alpha_{5}\right) \\
n_{2}= & k .
\end{aligned}
$$

Next, we will focus on the distribution of roots of the transcendental equation (4).

Lemma 1. For the transcendental equation ${ }^{[20]}$

$$
\begin{aligned}
& P\left(\lambda, \mathrm{e}^{-\lambda \tau_{1}}, \cdots, \mathrm{e}^{-\lambda \tau_{m}}\right)= \\
& \lambda^{n}+p_{1}^{(0)} \lambda^{n-1}+\cdots+p_{n-1}^{(0)} \lambda+p_{n}^{(0)}+ \\
& \quad\left[p_{1}^{(1)} \lambda^{n-1}+\cdots+p_{n-1}^{(1)} \lambda+p_{n}^{(1)}\right] \mathrm{e}^{-\lambda \tau_{1}}+\cdots+ \\
& \\
& {\left[p_{1}^{(m)} \lambda^{n-1}+\cdots+p_{n-1}^{(m)} \lambda+p_{n}^{(m)}\right] \mathrm{e}^{-\lambda \tau_{m}}=0}
\end{aligned}
$$

as $\left(\tau_{1}, \tau_{2}, \tau_{3}, \cdots, \tau_{m}\right)$ vary, the sum of orders of the zeros of $P\left(\lambda, \mathrm{e}^{-\lambda \tau_{1}}, \cdots, \mathrm{e}^{-\lambda \tau_{m}}\right)$ in the open right half plane can change, and only a zero appears on or crosses the imaginary axis.

When $\tau=0$, (4) becomes

$$
\lambda^{3}+\left(m_{2}+n_{2}\right) \lambda^{2}+\left(m_{1}+n_{1}\right) \lambda+m_{0}+n_{0}=0 .
$$

All roots of (5) have a negative real part if the following conditions hold

$m_{2}+n_{2}>0, m_{0}+n_{0}>0,\left(m_{2}+n_{2}\right)\left(m_{1}+n_{1}\right)>m_{0}+n_{0}$

Then the equilibrium point $E_{1}\left(x_{1}^{*}, y_{1}^{*}, z_{1}^{*}\right)$ is locally asymptotically stable when the conditions (H1) and (H2) hold.

For $\omega>0$, i $\omega$ is a root of (4) if and only if

$-\omega^{3} \mathrm{i}-m_{2} \omega^{2}+m_{1} \omega \mathrm{i}+m_{0}+\left(-n_{2} \omega^{2}+n_{1} \omega \mathrm{i}+n_{0}\right) \mathrm{e}^{-\omega \tau \mathrm{i}}=0$.

Separating the real and imaginary parts gives

$$
\left\{\begin{array}{l}
\left(n_{0}-n_{2} \omega^{2}\right) \cos (\omega \tau)+n_{1} \omega \sin (\omega \tau)=m_{2} \omega^{2}-m_{0} \\
n_{1} \omega \cos (\omega \tau)-\left(n_{0}-n_{2} \omega^{2}\right) \sin (\omega \tau)=\omega^{3}-m_{1} \omega .
\end{array}\right.
$$

It follows from (6) that

$$
\left(n_{0}-b_{2} \omega^{2}\right)^{2}+\left(n_{1} \omega\right)^{2}=\left(m_{2} \omega^{2}-m_{0}\right)^{2}+\left(\omega^{3}-m_{1} \omega\right)^{2}
$$

which is equivalent to

$$
\omega^{6}+p \omega^{4}+q \omega^{2}+r=0
$$

where

$$
p=m_{2}^{2}-n_{2}^{2}-2 m_{1}, q=m_{1}^{2}-2 m_{0} m_{2}+2 n_{0} n_{2}-n_{1}^{2}, r=m_{0}^{2}-n_{0}^{2} .
$$


Denote $z=\omega^{2}$, then (7) becomes

$$
z^{3}+p z^{2}+q z+r=0
$$

Let

$$
h(z)=z^{3}+p z^{2}+q z+r .
$$

We shall employ the ideas of $[21-23]$ to investigate the distribution of zeros of (4). We assume that

$$
\left|m_{0}\right|<\left|n_{0}\right| \text {. }
$$

Since $\lim _{t \rightarrow+\infty} h(z)=+\infty$ and $h(0)=r=m_{0}^{2}-n_{0}^{2}<0$, then (8) has at least one positive real root. Without loss of generality, we assume that (8) has three positive roots, denoted by $\omega_{k}(k=1,2,3)$. Then by $(6)$, we derive

$$
\tau_{k}^{(j)}=\frac{1}{\omega_{k}}(\arccos \theta+2 j \pi)
$$

where $k=1,2,3 ; j=0,1, \cdots$, and

$$
\theta=\frac{\left(m_{2} \omega^{2}-m_{0}\right)\left(n_{0}-n_{2} \omega_{k}^{2}\right)+n_{1} \omega_{k}\left(\omega_{k}^{3}-m_{1} \omega_{k}\right)}{\left(n_{0}-n_{2} \omega_{k}^{2}\right)^{2}+\left(n_{1} \omega_{k}\right)^{2}} .
$$

Then $\pm \omega_{k}$ i is a pair of purely imaginary roots of (4) when $\tau=\tau_{k}^{(j)}$. Define

$$
\tau_{0}=\tau_{k_{0}}^{(0)}=\min _{k \in\{1,2,3\}}\left\{\tau_{k}^{(0)}\right\} .
$$

The above analysis leads to the Lemma as follows.

Lemma 2. If (H1-H3) hold, then all roots of (4) have negative real parts when $\tau \in\left[0, \tau_{0}\right)$, and (4) admits a pair of purely imaginary roots $\pm \omega_{k}$ i when $\tau=\tau_{k}^{(j)}(k=1,2,3 ; j=$ $0,1,2, \cdots)$.

Let $\lambda(\tau)=\alpha(\tau)+\mathrm{i} \omega(\tau)$ be a root of (4) near $\tau=\tau_{k}^{(j)}$, and $\alpha\left(\tau_{k}^{(j)}\right)=0$, and $\omega\left(\tau_{k}^{(j)}\right)=\omega_{k}$. Due to functional differential equation theory, for every $\tau_{k}^{(j)}, k=1,2,3 ; j=0,1,2, \cdots$, there exists $\varepsilon>0$ such that $\lambda(\tau)$ is continuously differentiable in $\tau$ for $\left|\tau-\tau_{k}^{(j)}\right|<\varepsilon$. Substituting $\lambda(\tau)$ into the left hand side of (4) and taking derivative with respect to $\tau$, we have

$$
\begin{aligned}
{\left[\frac{\mathrm{d} \lambda}{\mathrm{d} \tau}\right]^{-1}=} & \frac{3 \lambda^{2}+2 m_{2} \lambda+m_{1}+\left(2 n_{2} \lambda+n_{1}\right) \mathrm{e}^{-\lambda \tau}}{\lambda\left(n_{2} \lambda^{2}+n_{1} \lambda+n_{0}\right) \mathrm{e}^{-\lambda \tau}}-\frac{\tau}{\lambda}= \\
& \frac{\left(3 \lambda^{2}+2 m_{2} \lambda+m_{1}\right) \mathrm{e}^{\lambda \tau}+\left(2 n_{2} \lambda+n_{1}\right)}{\lambda\left(n_{2} \lambda^{2}+n_{1} \lambda+n_{0}\right)}-\frac{\tau}{\lambda}
\end{aligned}
$$

Then

$$
\begin{aligned}
& {\left[\frac{\mathrm{d}(\operatorname{Re} \lambda(\tau))}{\mathrm{d} \tau}\right]_{\tau=\tau_{k}^{(j)}}^{-1}=} \\
& \operatorname{Re}\left\{\frac{\left(3 \lambda^{2}+2 m_{2} \lambda+a_{1}\right) \mathrm{e}^{\lambda \tau}+\left(2 n_{2} \lambda+n_{1}\right)}{\lambda\left(n_{2} \lambda^{2}+n_{1} \lambda+n_{0}\right)}\right\}_{\tau=\tau_{k}^{(j)}}= \\
& \operatorname{Re}\left\{\frac{\delta+2 n_{2} \omega_{k} \mathrm{i}+n_{1}}{\omega_{k} \mathrm{i}\left(-n_{2} \omega_{k}^{2}+n_{1} \omega_{k} \mathrm{i}+n_{0}\right)}\right\}= \\
& \operatorname{Re}\left\{\frac{\theta_{1}+\theta_{2} \mathrm{i}}{\theta_{3}+\theta_{4} \mathrm{i}}\right\}=\frac{\theta_{1} \theta_{3}+\theta_{2} \theta_{4}}{\theta_{3}^{2}+\theta_{4}^{2}}
\end{aligned}
$$

where

$$
\delta=\left(-3 \omega_{k}^{2}+2 m_{2} \omega_{k} \mathrm{i}+m_{1}\right)\left(\cos \omega_{k} \tau_{k}^{(j)}+i \sin \omega_{k} \tau_{k}^{(j)}\right)
$$

and

$$
\begin{aligned}
& \theta_{1}=\left(m_{1}-3 \omega_{k}^{2}\right) \cos \omega_{k} \tau_{k}^{(j)}-2 m_{2} \omega_{k} \sin \omega_{k} \tau_{k}^{(j)}+n_{1} \\
& \theta_{2}=2 m_{2} \omega_{k} \cos \omega_{k} \tau_{k}^{(j)}+\left(m_{1}-3 \omega_{k}^{2}\right) \sin \omega_{k} \tau_{k}^{(j)}+2 n_{2} \omega_{k} \\
& \theta_{3}=-n_{1} \omega_{k}^{2} \\
& \theta_{4}=\omega_{k}\left(m_{0}-n_{2} \omega_{k}^{2}\right) .
\end{aligned}
$$

We assume that the following condition holds.

$$
\theta_{1} \theta_{3}+\theta_{2} \theta_{4} \neq 0
$$

According to above analysis and the results of Kuang ${ }^{[24]}$ and Hale ${ }^{[25]}$, we have Theorem 1.

Theorem 1. If (H1) and (H3) hold, then the equilibrium $E\left(x_{1}^{*}, y_{1}^{*}, z_{1}^{*}\right)$ of system (2) is asymptotically stable for $\tau \in\left[0, \tau_{0}\right)$. Under the conditions (H1-H3), if the condition (H4) holds, then system (2) undergoes a Hopf bifurcation at the equilibrium $E\left(x_{1}^{*}, y_{1}^{*}, z_{1}^{*}\right)$ when $\tau=\tau_{k}^{(j)}, k=1,2,3 ; j=$ $0,1,2, \cdots$.

\section{Numerical simulations}

In this section, we now carry out some numerical simulations work (using Matlab dde23) to verify the analytical predictions obtained in the previous section. As an example, we consider the following delayed feedback control system with the parameters $\alpha_{1}=30, k_{-1}=0.415, \alpha_{5}=$ $10, \alpha_{4}=16.5, k_{-5}=0.5$, then system (2) becomes

$$
\left\{\begin{aligned}
\dot{x}_{1}= & 30 x_{1}-0.415 x_{1}^{2}-x_{1} y_{1}-x_{1} z_{1}+ \\
& k\left[x_{1}-x_{1}(t-\tau)\right] \\
\dot{y}_{1}= & x_{1} y_{1}-10 y_{1} \\
\dot{z}_{1}= & 16.5 z_{1}-x_{1} z_{1}-0.5 z_{1}^{2}
\end{aligned}\right.
$$

System (13) has a positive equilibrium $E(10,12.85,13)$. From Theorem 1, we know that when $k>9.35$, the characteristic (4) always has roots with positive real parts for all $\tau \geqslant 0$. Thus, for the purpose of controlling chaos, we consider $k<9.35$. In particular, we let $k=-8$. It is easy to obtain that $(\mathrm{H} 1-\mathrm{H} 3)$ are satisfied. Take $j=0$ for example, by some computation by means of Matlab 7.0 , we get $\omega_{0} \approx 0.4478, \tau_{0} \approx 0.6$. Thus the equilibrium $E(10,12.85,13)$ is asymptotically stable for $\tau<\tau_{0} \approx 0.6$ and unstable for $\tau>\tau_{0} \approx 0.6$ which is shown in Fig. 2 . When $\tau=\tau_{0} \approx 0.6$, (13) undergoes a Hopf bifurcation at the equilibrium $E(10,12.85,13)$, i.e., a periodic solution of small amplitude occurs around $E(10,12.85,13)$ when $\tau$ is close to $\tau_{0}=0.6$ which can be seen in Fig. 3 . In addition, we find that the varying initial values have no effect on the stability and Hopf bifurcation behavior of system (13). 

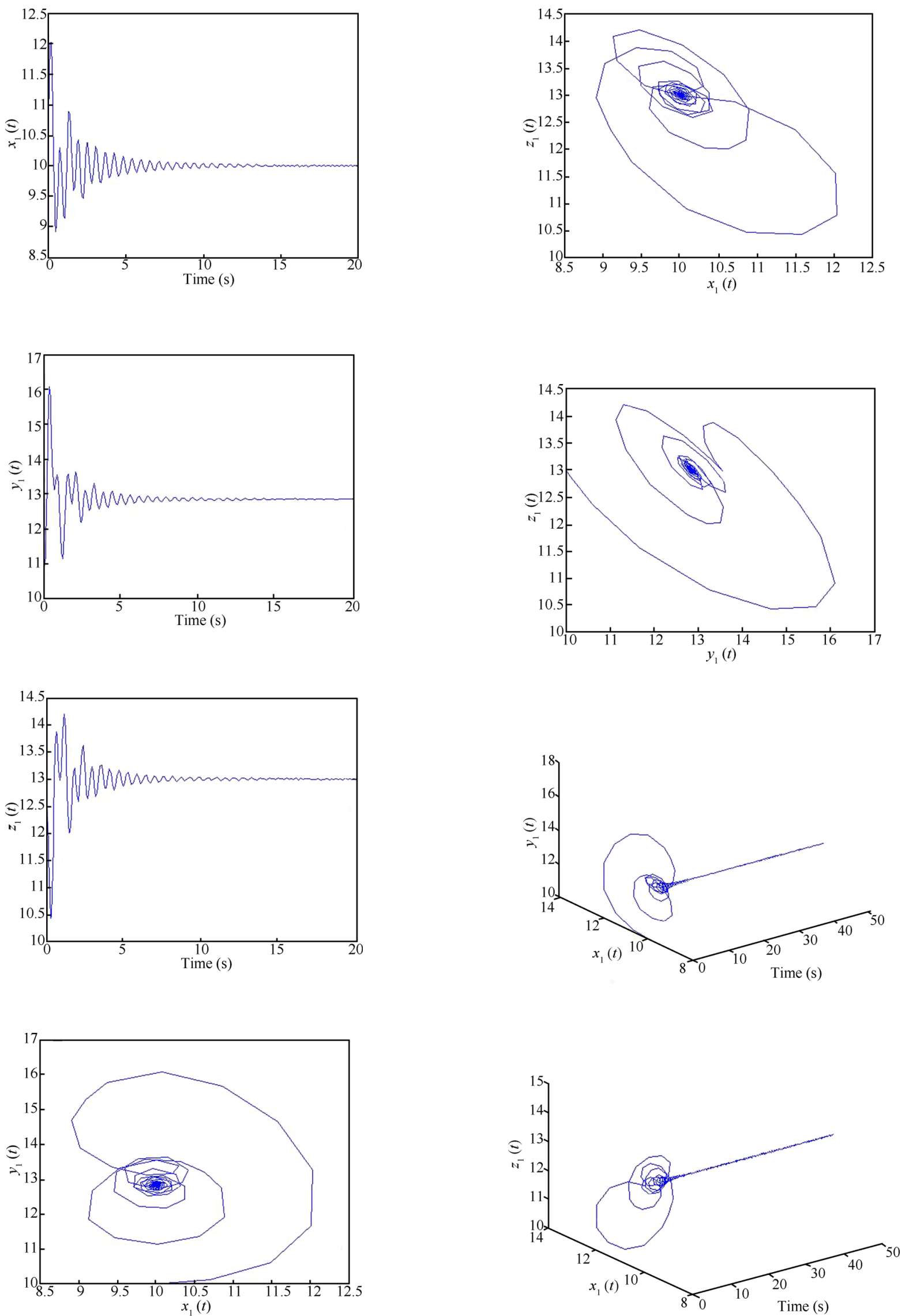

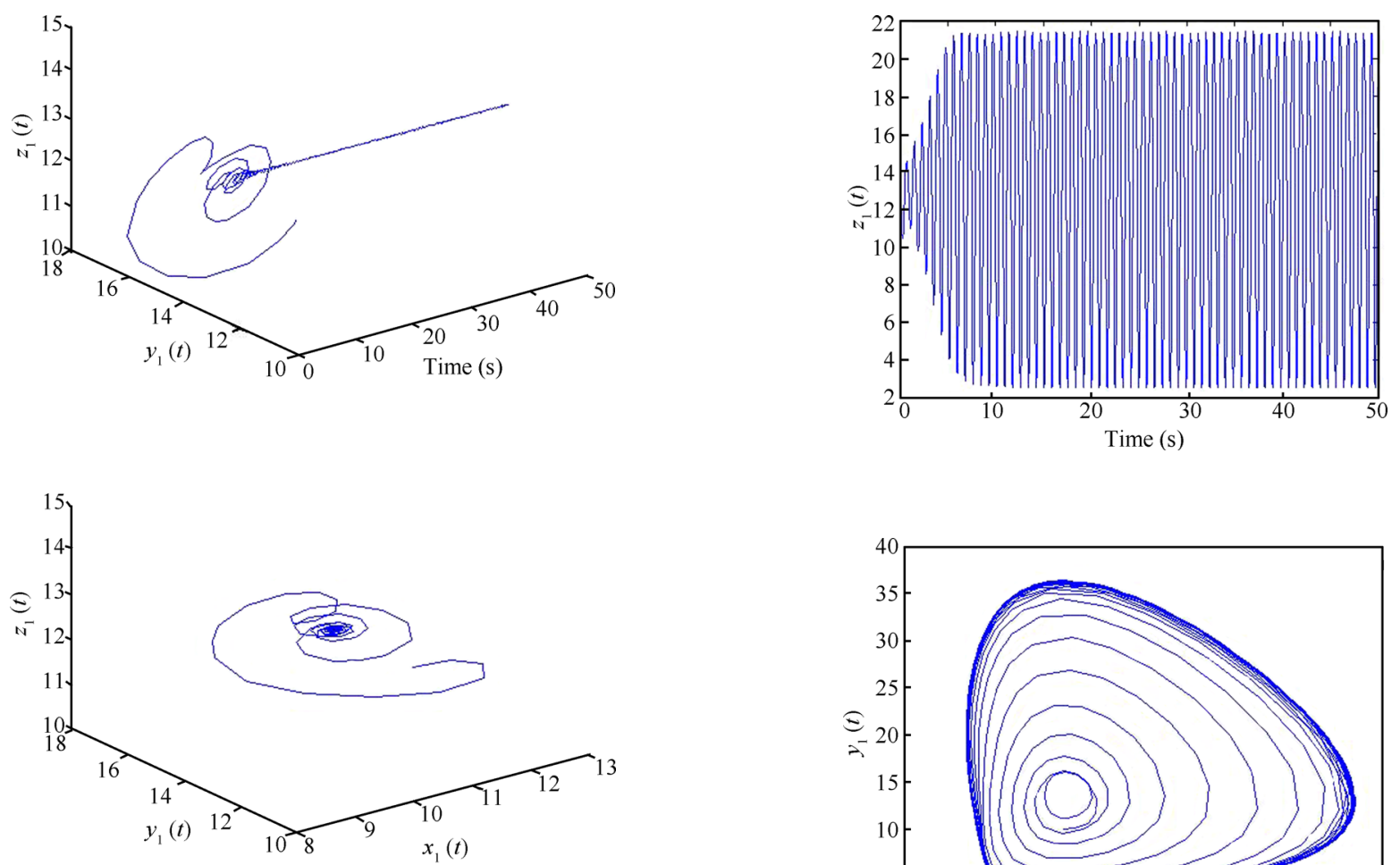

Fig. 2 Chaos vanishes when $\tau=0.5<\tau_{0} \approx 0.6$. The equilibrium $E(10,12.85,13)$ is asymptotically stable. The initial value is $(10,10,13)$
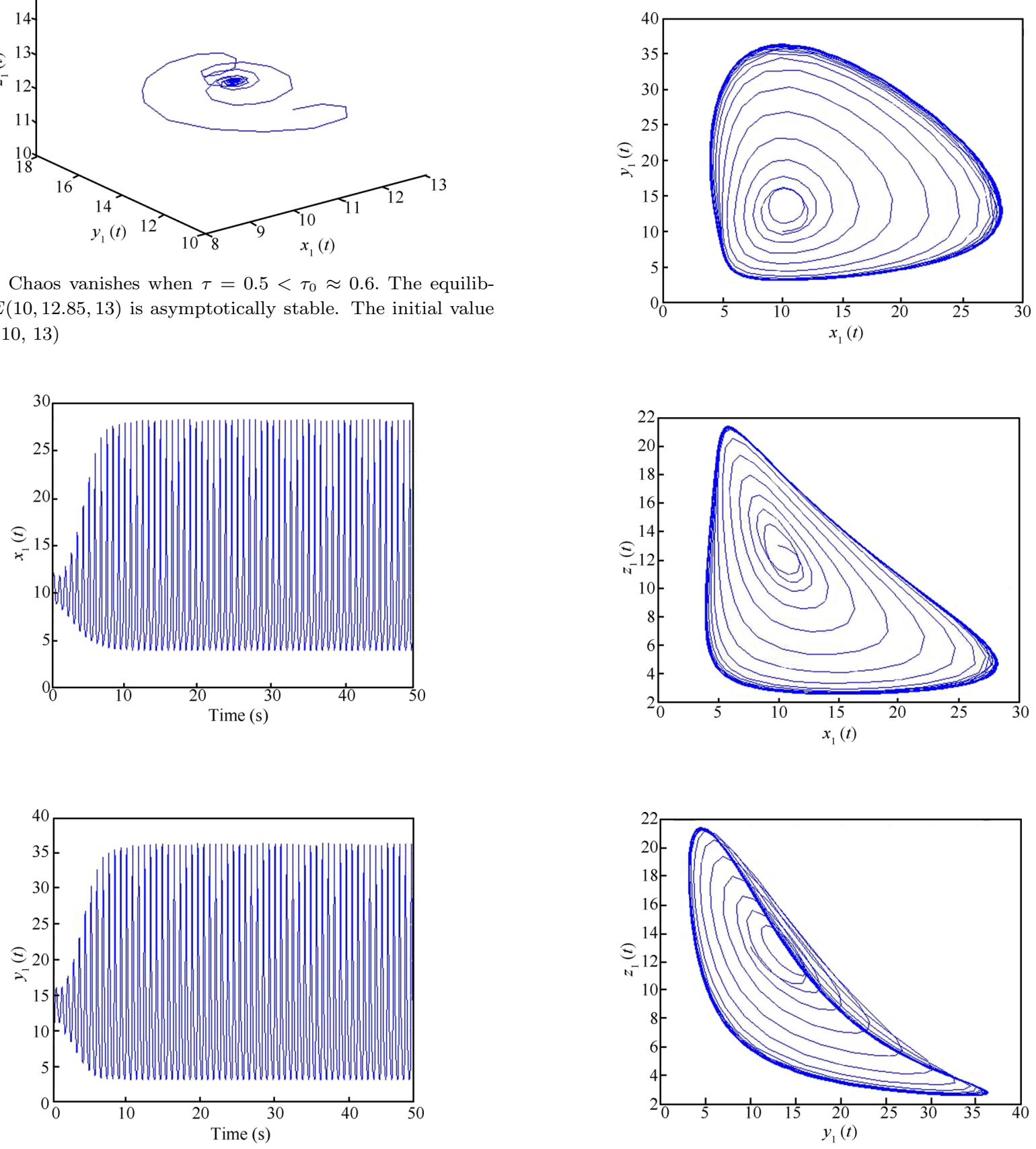

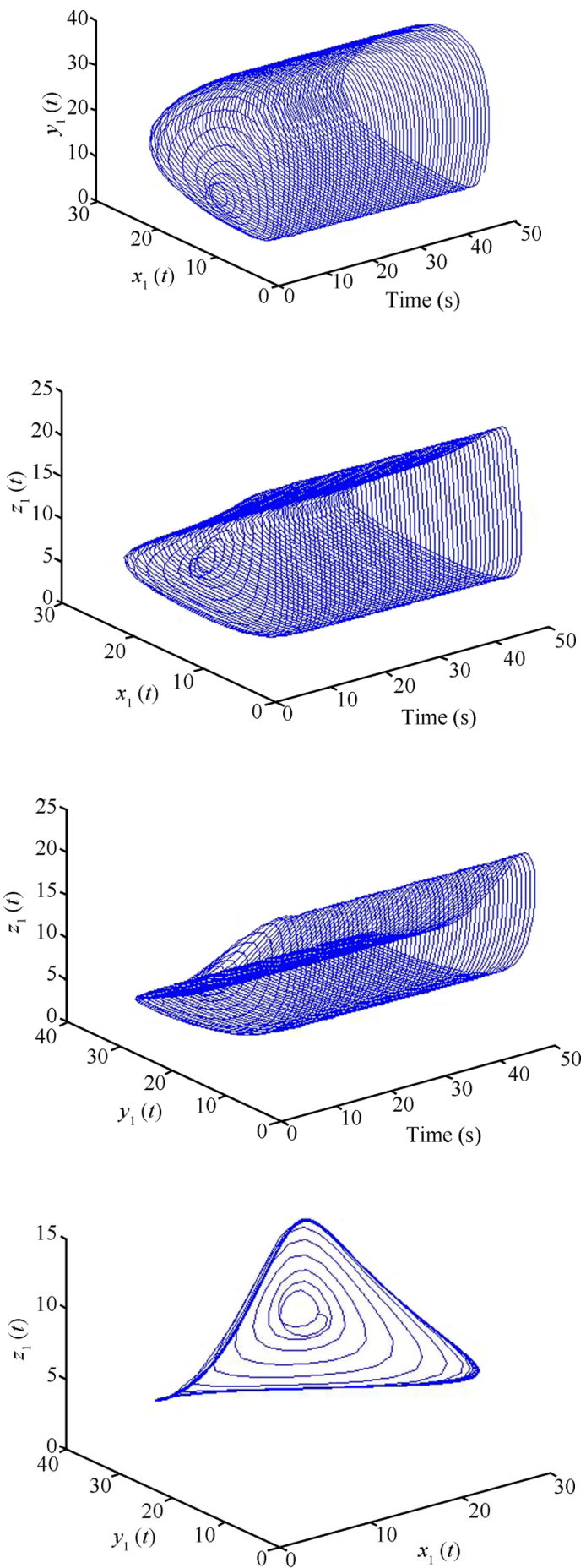

Fig. 3 Chaos vanishes when $\tau=0.8>\tau_{0} \approx 0.6$. Hopf bifurcation occurs from the equilibrium $E(10,12.85,13)$. The initial value is $(10,10,13)$

\section{Conclusions}

In this paper, a feedback control method is used to suppress chaotic behavior of chemical dynamical system within the chaotic attractor. By adding a time-delayed force to the first equation of the chemical system, we have investigated the local stability of the equilibrium $E\left(x_{1}^{*}, y_{1}^{*}, z_{1}^{*}\right)$ and local Hopf bifurcation of the delayed chemical model. It is showed that under the conditions (H1-H3), the chemical system is asymptotically stable for $\tau \in\left[0, \tau_{0}\right)$ and unstable for $\tau>\tau_{0}$. If $(\mathrm{H} 1-\mathrm{H} 4)$ are satisfied, a sequence of Hopf bifurcations occur near the equilibrium $E\left(x_{1}^{*}, y_{1}^{*}, z_{1}^{*}\right)$, i.e., a family of periodic orbits bifurcate from the the equilibrium $E\left(x_{1}^{*}, y_{1}^{*}, z_{1}^{*}\right)$. The obtained results show that chaos vanishes, and then chaos can be suppressed. Numerical simulations verifying our theoretical predictions are included.

\section{References}

[1] T. G. Gao, Z. Q. Chen, Z. Z. Yuan. Control for the synchronization of Chen system via a single nonlinear input. Journal of Control Theory and Applications, vol. 4, no. 3, pp. 297-301, 2006.

[2] G. R. Chen, X. N. Dong. From Chaos to Order Methodologies, Perspectives and Applications, Singapore: World Scientific, 1998.

[3] E. Ott, C. Grebogi, J. A. Yorke. Controlling chaos. Physical Review Letters, vol. 64, no. 11, pp. 1196-1199, 1990.

[4] Y. W. Wang, Z. H. Guan, H. O. Wang. Feedback and adaptive control for the synchronization of Chen system via a single variable. Physics Letters A, vol. 312, no. 1-2, pp. 34$40,2003$.

[5] G. R. Chen. Controlling Chaos and Bifurcations in Engineering Systems, Boca Raton, FL: CRC Press, 1999.

[6] M. Wan. Convergence and chaos analysis of a blind decorrelation neural network. Journal of Information and Computational Science, vol. 8, no. 5, pp. 791-798, 2011.

[7] Y. W. Deng, G. X. Sun, J. Q. E. Application of chaos optimization algorithm for robust controller design and simulation study. Journal of Information and Computational Science, vol. 7, no. 13, pp. 2897-2905, 2010.

[8] X. C. Shi, T. P. Zhang. Adaptive tracking control of uncertain MIMO nonlinear systems with time-varying delays and unmodeled dynamics. International Journal of Automation and Computing, vol. 10, no. 3, pp. 194-201, 2013.

[9] M. M. Zirkohi, M. M. Fateh, M. A. Shoorehdeli. Type-2 fuzzy control for a flexible-joint robot using voltage control strategy. International Journal of Automation and Computing, vol. 10, no. 3, pp. 242-255, 2013.

[10] E. W. Bai, K. E. Lonngren. Sequential synchronization of two Lorenz systems using active control. Chaos, Solitons and Fractals, vol. 11, no. 7, pp. 1041-1044, 2000.

[11] X. S. Yang, G. R. Chen. Some observer-based criteria for discrete-time generalized chaos synchronization. Chaos, Solitons and Fractals, vol. 13, no. 6, pp. 1303-1308, 2002.

[12] G. R. Chen, X. Dong. On feedback control of chaotic continuous-time systems. IEEE Transactions on Circuits and Systems I: Fundamental Theory and Applications, vol. 40, no. 9, pp. 591-601, 1993.

[13] M. T. Yassen. Chaos control of Chen chaotic dynamical system. Chaos, Solitons and Fractals, vol. 15, no. 2, pp. 271283, 2003. 
[14] H. N. Agiza. Controlling chaos for the dynamical system of coupled dynamos. Chaos, Solitons and Fractals, vol. 13, no. 2, pp. 341-352, 2002.

[15] E. N. Sanchez, J. P. Perez, M. Martinez, G. R. Chen. Chaos stabilization: An inverse optimal control approach. Latin American Applied Research, vol. 32, no. 1, pp. 111$114,2002$.

[16] M. T. Yassen. Adaptive control and synchronization of a modified Chuas circuit system. Applied Mathematics and Computation, vol. 135, no. 1, pp.113-128, 2001.

[17] T. L. Liao, S. H. Lin. Adaptive control and synchronization of Lorenz systems. Journal of the Franklin Institute, vol. 336, no. 6, pp. 925-937, 1999.

[18] Y. Huang, X. S. Yang. Chaoticity of some chemical attractors: A computer assisted proof. Journal of Mathematical Chemistry, vol. 38, no. 1, pp. 107-117, 2005.

[19] K. Pyragas. Continuous control of chaos by self-controlling feedback. Physics Letters A, vol.170, no. 6, pp. 421-428, 1992.

[20] S. G. Ruan, J. J. Wei. On the zeros of a third degree exponential polynomial with applications to a delayed model for the control of testosterone secretion. Mathematical Medicine and Biology, vol. 18, no. 1, pp. 41-52, 2001.

[21] X. L. Li, J. J. Wei. On the zeros of a fourth degree exponential polynomial with applications to a neural network model with delays. Chaos, Solitons and Fractals, vol. 26, no. 2, pp. 519-526, 2005.

[22] Y. L. Song, J. J. Wei. Bifurcation analysis for Chens system with delayed feedback and its application to control of chaos. Chaos, Solitons and Fractals, vol. 22, no. 1, pp. 7591, 2004.

[23] Y. Yang. Hopf bifurcation in a two-competitor, one-prey system with time delay. Applied Mathematics and Computation, vol. 214, no. 1, pp. 228-235, 2009.

[24] Y. Kuang. Delay Differential Equations: With Applications in Population Dynamics, San Diego: Academic Press, Incorporated, 1993.

[25] J. K. Hale. Theory of Functional Differential Equation, Berlin: Springer-Verlag, 1977.

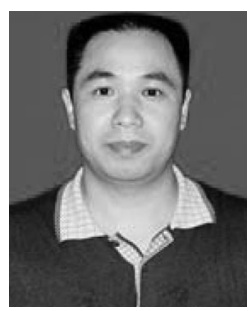

pers.

His research interests include stability and bifurcation theory of delayed differential equation.

E-mail: xcj403@126.com (Corresponding author)

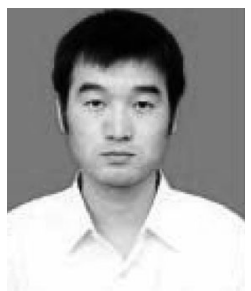

Yu-Sen Wu graduated from Liaocheng University, China in 2004. He received his M. Sc. degree from Central South University, China in 2007, and his Ph. D. degree from Central South University, China in 2010. He is currently an associate professor at School of Mathematics and Statistics of Henan University of Science and Technology. He has published about 30 refereed journal papers.

His research interests include the qualitative theory of ordinary differential equation and computer symbol calculation.

E-mail: wuyusen621@126.com 